\title{
Bevacizumab in small cell lung cancer
}

\author{
Giandomenico Roviello ${ }^{1,2}$, Navid Sobhani ${ }^{2}$, Daniele Generali ${ }^{2,3}$ \\ ${ }^{1}$ Unit of Medical Oncology, Department of Oncology, San Donato Hospital, Arezzo, Italy; ${ }^{2}$ Department of Medical, Surgery and Health Sciences, \\ University of Trieste, Trieste, Italy; ${ }^{3}$ Unit of Molecular Therapy and Pharmacogenomic, ASST Cremona, Cremona, Italy \\ Correspondence to: Giandomenico Roviello, MD. Unit of Medical Oncology, Department of Oncology, Ospedale San Donato, Arezzo 52100, Italy. \\ Email: giandomenicoroviello@gmail.com. \\ Provenance: This is a Guest Editorial commissioned by Section Editor Jianrong Zhang, MD (Department of Thoracic Surgery, First Affiliated \\ Hospital of Guangzhou Medical University, Guangzhou Institute of Respiratory Disease, Guangzhou, China). \\ Comment on: Tiseo M, Boni L, Ambrosio F, et al. Italian, Multicenter, Phase III, Randomized Study of Cisplatin Plus Etoposide With or Without \\ Bevacizumab as First-Line Treatment in Extensive-Disease Small-Cell Lung Cancer: The GOIRC-AIFA FARM6PMFJM Trial. J Clin Oncol \\ 2017;35:1281-7.
}

Submitted May 16, 2017. Accepted for publication Jun 01, 2017.

doi: 10.21037/atm.2017.06.44

View this article at: http://dx.doi.org/10.21037/atm.2017.06.44

The randomized phase III, open-label, multicenter clinical trial, based on 205 patients with extensive-disease (ED) of small-cell lung cancer (SCLC), evaluated the efficacy and the safety of humanized monoclonal antibody targeting vascular endothelial growth factor (VEGF), in combination with etoposide and cisplatin in first line chemotherapeutic treatment (1). This study was supported by the "Gruppo Oncologico Italiano di Ricerca Clinica (GOIRC)Agenzia Italiana del Farmaco FARM6PMFJM”. A random assignment was made for the patients to either receive cisplatin and etoposide with or without bevacizumab. The evaluation of the overall survival (OS) was the primary end point. In the bevacizumab arm, at a median follow-up of 34.9 months, there was an improvement in the median OS (9.8 vs. 8.9 months; HR =0.78) showing, respectively, a 1 -year survival rates of $37 \%$ and $25 \%$ ( $\mathrm{HR}=0.78 ; 95 \%$ CI, 0.58-1.06; $\mathrm{P}=0.113$ ). Moreover, patients reporting an objective response were in proportion $58.4 \%$ and $55.3 \%$ for the bevacizumab and the control arms, respectively. Ninety six patients were treated with chemotherapy and bevacizumab and 41 of them (42\%) continued with the monoclonal antibody beyond the pre-planned six cycles of treatment with maintenance, in median, of four more cycles. The disease progression caused the interruption of bevacizumab in $65.8 \%$ of patients. The bevacizumabbased maintenance had a statistically significant effect over the OS ( $\mathrm{HR}=0.60 ; \mathrm{P}=0.011$ ) with, as expected, a welltolerability; the only frequent adverse registered event in the bevacizumab arm was the hypertension (grade 3 or 4 ; $6.3 \%$ vs. $1.0 \% ; \mathrm{P}=0.057)$.

In several different tumours, such as non-small cell lung cancer (NSCLC) (2-5), the effect of the anti-angiogenic antibody on the survival was reported. In NSCLC, bevacizumab is now approved in combination with the standard platinum based chemotherapy or as maintenance after chemotherapy, for the treatment of NSCLC patients without driver-mutations (3-5). On the counterpart, in SCLC, the randomized trials using bevacizumab added to standard chemotherapy showed poor results (6).

In 2011, a randomized phase II trial based on chemotherapy with bevacizumab versus chemotherapy plus placebo in previously untreated extensive-stage disease-SCLC (SALUTE) (7) has been performed. The progression-free survival (PFS), indicated as the primary end-point, was met; however, the advantage of 5.5 months in the bevacizumab-based arm versus the 4.4 months in the placebo-based arm was not correlated with an increased OS, which remained longer in the control arm (9.4 vs. 10.9 months). The IFCT-0802 study confirmed this trend, failing to show that bevacizumab-based treatment was able to improve the survival (8).

The SCLC is considered a highly proliferating disease with higher tumor response rates; thus, the maintenance approach aiming to prolong the initial response to the standard chemotherapy is strongly supported due to its high tumor spreading nature. Targeted therapies, such 
as bevacizumab, with a good toxicity profiling could be administered for longer periods and are considered an ideal approach for the maintenance treatment. However, the use of new targeted therapies as maintenance compared to placebo control did not showed any advantage in term of survival as dispatched in the recent meta-analysis on SCLC (9). In contrast, Tiseo et al. showed patients receiving maintenance-based bevacizumab had a significantly improved OS (1). Considering the possible bias related to only patients who did not progress after chemotherapy accessed to maintenance-based bevacizumab, in our opinion, future randomized trials with specified endpoints should investigate the issue of bevacizumab-based maintenance approach in the management of patients with SCLC who respond to first line therapy.

It is also noteworthy, there is a lack of validated predictive factors that could foresee a response to bevacizumab (8,10-12); notwithstanding this fact, there are few clinical reports suggesting that hypertension has a potential role in defining responders versus no-responders to the VEGFtargeting monoclonal antibody (13). A pre-planned subgroup analysis reported by Tiseo et al. (1) indicated that sex, in favour to females, is a potential interactor with bevacizumab, but without providing exhaustive indications or explanations. Therefore, the issue of clinical or biological markers predictive of response to bevacizumab in SCLC is unresolved and future prospective trials on this topic are awaited. In the era of immunotherapy, it should be noted SCLC has a high immunogenic biological profile, partially due to the loads of mutations, supporting the newly encouraging results using specific immunotherapeutic approaches, like checkpoint inhibitors, capable of arousing the immune system against this cancer (14). In this scenario, it would be interesting to further investigate how immunotherapy in combination with bevacizumab-based maintenance and chemotherapeutic regimen would improve the OS in SCLC. There is, therefore, a strong rationale to support a multi-directional therapeutic intervention as a valid alternative approach for the SCLC management.

To conclude, in SCLC, the addition of bevacizumab to standard first-line platinum-etoposide based chemotherapy did not show an increase on patients' survival. Considering the report by Tiseo et al., there is a clinical need to test new biological drug based maintenance approach in SCLC. Furthermore, in our viewpoint, new clinical trials on maintenance therapy in SCLC should also clearly consider potential surrogate biomarkers predictive of response/ resistance in order to achieve a better selection of candidates who will have benefit to the therapy based on single or multi-directional biological drug-based maintenance treatment.

\section{Acknowledgements}

None.

\section{Footnote}

Conflicts of Interest: The authors have no conflicts of interest to declare.

\section{References}

1. Tiseo M, Boni L, Ambrosio F, et al. Italian, Multicenter, Phase III, Randomized Study of Cisplatin Plus Etoposide With or Without Bevacizumab as First-Line Treatment in Extensive-Disease Small-Cell Lung Cancer: The GOIRCAIFA FARM6PMFJM Trial. J Clin Oncol 2017;35:1281-7.

2. Roviello G, Bachelot T, Hudis CA, et al. The role of bevacizumab in solid tumours: A literature based meta-analysis of randomised trials. Eur J Cancer 2017;75:245-58.

3. Sandler A, Gray R, Perry MC, et al. Paclitaxel-carboplatin alone or with bevacizumab for non-small-cell lung cancer. N Engl J Med 2006;355:2542-50.

4. Reck M, von Pawel J, Zatloukal P, et al. Phase III trial of cisplatin plus gemcitabine with either placebo or bevacizumab as first-line therapy for nonsquamous non-small-cell lung cancer: AVAil. J Clin Oncol 2009;27:1227-34.

5. Petrioli R, Francini E, Fiaschi AI, et al. Switch maintenance treatment with oral vinorelbine and bevacizumab after induction chemotherapy with cisplatin, gemcitabine and bevacizumab in patients with advanced non-squamous non-small cell lung cancer: a phase II study. Med Oncol 2015;32:134.

6. Roviello G, Generali D. Is there a Place for Bevacizumab in Patients with Extensive-Stage Small Cell Lung Cancer? Curr Cancer Drug Targets 2016;16:209-14.

7. Spigel DR, Townley PM, Waterhouse DM, et al. Randomized phase II study of bevacizumab in combination with chemotherapy in previously untreated extensive-stage small-cell lung cancer: results from the SALUTE trial. J Clin Oncol 2011;29:2215-22.

8. Pujol JL, Lavole A, Quoix E, et al. Randomized phase II-III study of bevacizumab in combination with 
chemotherapy in previously untreated extensive small-cell lung cancer: results from the IFCT-0802 trial†. Ann Oncol 2015;26:908-14.

9. Roviello G, Zanotti L, Cappelletti MR, et al. No Advantage in Survival With Targeted Therapies as Maintenance in Patients With Limited and ExtensiveStage Small Cell Lung Cancer: A Literature-Based Meta-Analysis of Randomized Trials. Clin Lung Cancer 2016;17:334-40.

10. Petrioli R, Roviello G, Laera L, et al. Cisplatin, Etoposide, and Bevacizumab Regimen Followed by Oral Etoposide and Bevacizumab Maintenance Treatment in Patients With Extensive-Stage Small Cell Lung Cancer: A Single-Institution Experience. Clin Lung Cancer 2015;16:e229-34.

11. Jalal S, Bedano P, Einhorn L, et al. Paclitaxel plus

Cite this article as: Roviello G, Sobhani N, Generali D. Bevacizumab in small cell lung cancer. Ann Transl Med 2017;5(17):361. doi: 10.21037/atm.2017.06.44 bevacizumab in patients with chemosensitive relapsed small cell lung cancer: a safety, feasibility, and efficacy study from the Hoosier Oncology Group. J Thorac Oncol 2010;5:2008-11.

12. Horn L, Dahlberg SE, Sandler AB, et al. Phase II study of cisplatin plus etoposide and bevacizumab for previously untreated, extensive-stage small-cell lung cancer: Eastern Cooperative Oncology Group Study E3501. J Clin Oncol 2009;27:6006-11.

13. Dionísio de Sousa IJ, Ferreira J, Rodrigues J, et al. Association between bevacizumab-related hypertension and response to treatment in patients with metastatic colorectal cancer. ESMO Open 2016;1:e000045.

14. Tartarone A, Giordano P, Lerose R, et al. Progress and challenges in the treatment of small cell lung cancer. Med Oncol 2017;34:110. 